\title{
THE ROLE OF FILAMENT CURRENTS IN A FLARE ACTIVE REGION
}

\author{
XU AOAO \\ WU GUIPING ${ }^{2}$, TANG YUHUA ${ }^{1}$, JANG ZHIBO ${ }^{3}$ \\ 1. Department of Astronomy, Nanjing University \\ 2. Physics Teaching Researching, Nanjing Railway Medical College \\ 3. Purple Mountain Observatory, Chinese Academy of Sciences
}

\section{INTRODUCTION}

The two-ribbon flare of May 13, 1981 is one of the most notable flares during solar cycle 21 . There are rich observational data of this flare. We study carefully the characteristics about the evolution and motion of the filament related with the flare as well as the relationship between these characteristics and the development of the flare. The results indicate that the increase of the filament current plays an important role in the occurrence and development of the flare.

\section{THE IMPORTANT OBSERVATIONAL RESULTS}

In order to explain theoretically the flare of May 13, 1981, we have to notice the following important observational results.

(1) There is a clear boundary between thermal and non-thermal phases of the flare. Moreover, the flash phase of the flare occurred before the impulsive phase.

(2) Before and during the flare, the east-segment of the filament related with the flare had apparently risen. (see Figure 1)

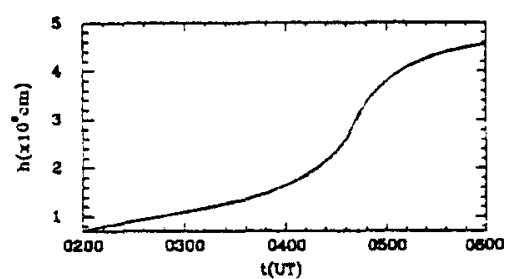

Fig.1 The time profile of height of the filament

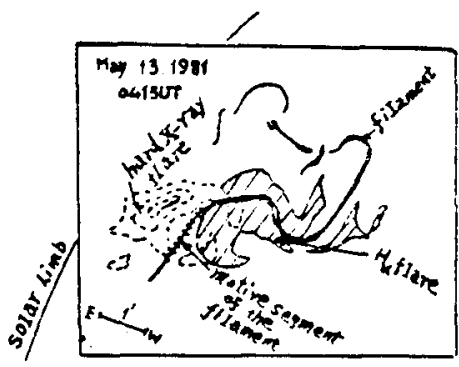

Fig.2 The Flare of May 13,1961

(3) The position of the X-ray source of the flare was basically consistent with the east-segment of the filament. Furthermore, the $X$-ray source extended along the ES-WN direction as the east-segment of the filament was directed. (see Figure.2) 

(FLASH PHASE) OF THE FLARE OF MAY 13, 1981

1. The ascending motion of the filament implies the increase of the filament current (Wu and $\mathrm{Xu}, 1991$ ). Accoridng to Kuperus-Raadu filament model, (Kuperus and Raadu, 1974), the equation of motion of the filaments in a background field is $(\mathrm{Xu}$, 1991)

$$
M \frac{d^{2} h}{d t^{2}}=L \frac{I^{2}}{c^{2} h}-L \frac{I B}{c}-\frac{M g_{\odot} R_{\odot}^{2}}{\left(R_{\odot}+h\right)^{2}}
$$

where $B$ is the strength of the background potential field, $L$ and $M$ are the length and mass of the filament respectively. We solve the equation (1) in terms of $h-t$ curve and obtain the I-t curve (see Figure 3). Figure 3 shows the current intensity of the filament increases with time.

2. According to the observation and theoretical analysis, we can apply the Lundquist field to describe the magnetic field inside the filament. Thus, the relation between the current intensity of the filament and magnetic shear within the filament is obtained $(\mathrm{Xu}, 1992)$ :

$$
I=\frac{c B_{1} R}{2} J_{1}(\alpha B)
$$

where $\alpha$ is the force-free factor as the measure of magnetic shear, $B_{1}$ is the magnetic strength at the axis of cylinder of the filament, $R$ is the radius of the filament, $J_{1}$ is the first order of Bessel function.

Using equation (2) and Figure 3, we can get $\alpha(t)$ which increases with time too.

3. The tearing mode instability within the filament can convert the magnetic energy in to thermal energy of the filament and flare region. The energy equation of the filament plasma is (Cao et al., 1986)

$$
\frac{R_{c}}{y-1} \frac{d T_{c}}{d t}=\frac{d Q}{d t}=\chi_{1} n_{c}^{2} T_{\infty}^{\alpha_{1}}-\chi_{2} n_{c}^{2} T_{c}^{\alpha_{2}^{2}}+\varepsilon_{0} e^{2 \gamma_{c} t}+\kappa_{\sigma} T_{c}^{3 / 2}\left(T_{\infty}-T_{c}\right) / L^{2}
$$

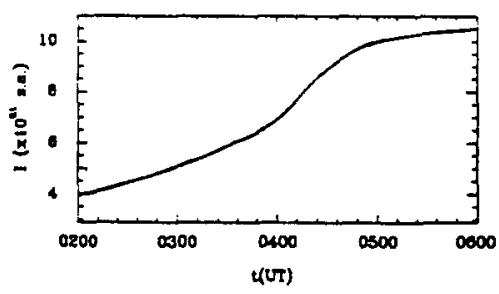

Fig.3 The time profile of current intensity of the filament

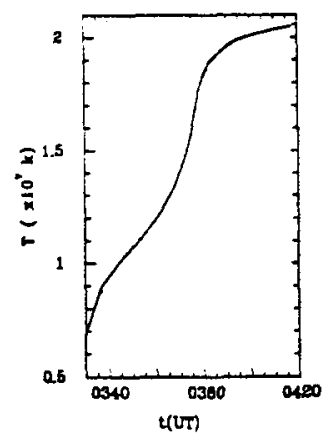

Fig.4 The time profile of the temperature in the region of the flare sources

According to the observed values of physical parameters of the flare and the 
growth rate $\gamma_{C}$ of the tearing mode instability, we solve equation (3) and obtaine Tc-t curve (see Figure 4). The change of Tc coincides with the observation data of the microwave radiation (Tsuneta, 1983)

\section{THE PHYSICAL MECHANISM OF NON-THERMAL PHASE (IM- PULSIVE PHASE) OF THE FLARE OF MAY 13, 1981}

The observations indicate that the flare clearly exhibits non-thermal characteristics after $0412 \mathrm{UT}$. We are very interested in the following observational data:

(1) The microwave source and hard X-ray source both located at the top of the flare loop.

(2) There is a very broad population of non-thermal electrons.

(3) The electron temperature doesn't change during the non-thermal phase.

(4) The II -type radio burst was not observed and the position of the hard $\mathrm{X}$-ray source is stable during the flare. These observational results show that non-thermal electrons couldn't be accelerated by $\Pi$-type shock wave.

(5) The growth rate of the filament current at 040SUT became suddenly large and was 3 times the one before $0405 \mathrm{UT}$ in Figure 3. It causes the strong current density occurring near the filament surface.

In terms of these observed results, we think that the accelerative process of the non-thermal electrons is describled by the revised Alfven-Carlqvist Model (Spicer, 1981). The Dreicer field is the accelerative mechanism of the non-thermal electrons in a thermal plasma. The production rate $\%_{0}$ of runaway electrons per volume can be calculated (Holman, 1985).

$$
\gamma_{0}=0.35 v_{\text {eif }}\left(v_{e} / v_{c}\right)^{3 / 4} \exp \left[-1.41\left(v_{0} / v_{c}\right)-0.25\left(v_{e} / v_{c}\right)^{2}\right]
$$

where $\mathrm{V}_{\bullet}=\left(\mathrm{kT}, / \mathrm{m}_{\bullet}\right)^{1 / 2}, \mathrm{~V}_{\mathrm{c}}=\left(\mathrm{m}_{\bullet} v_{\text {eff }} \mathrm{V}_{\bullet}^{3} / \mathrm{eE}\right)^{1 / 2}, v_{\text {eff }}$ is the effective collision frequence. Thus, the total number of the runaway electrons is:

$$
\mathrm{N}=\mathrm{n}_{0} \gamma_{0} 2 \pi \mathrm{RL} \Delta \mathrm{r}_{1} \Delta \mathrm{t}
$$

where $\Delta r$ is the width of the current sheath at the surface of the filament, $\Delta t$ is the duration of the non-thermal phase. Substituting the relevant parameters into equations.(5), we get

$$
\mathrm{N}=10^{35}-10^{36}
$$

The result is reasonable.

\section{REFERENCE}

Cao, Tianjun, et al., 1986 Acta Astronomica Sinica, 27,317.

Holman, G. D., 1985, Ap.J., 293, 584

Kuperus, M., and Raadu, M.A., 1974, Astron. Astrophys., 31, 189.

Spicer, D.S., 1981, Solar Physi., 70, 149.

Tsuneta, S., et al., 1983, Solar Phys., 86, 213.

Wu, S.T. and Xu, A.A., 1991, Eruptive Solar Flare, eds by Z. Svestka.

B.V, Jackson, M.E. Machado, 55.

Xu, Aoao, 1991, Acta Astronomica Sinica, 32, 343.

$\mathrm{Xu}, \mathrm{A}$ oao, 1992 Chinese Journai of Space Science, 12, 1. 